\title{
A REDUCED TABLEOF THE ZECH'S LOGARITHM
}

\author{
O. C. Justiz' ${ }^{1}$, E. M. Capó ${ }^{2}$, P. F. Arrozarena ${ }^{3}$, G. S. Gómez ${ }^{4}$ \\ Departament of Mathematics. Central University "Marta Abreu" of Las Villas. Cuba \\ oristela@uclv.edu.cu \\ Departament of Mathematics. Central University "Marta Abreu" of Las Villas. Cuba \\ evaristoj@uclv.cu \\ Habana University. Cuba \\ pfreyre@matcom.uh.cu \\ Center ofMathematics Research. Guanajuato. México \\ guillermo.sosa@cimat.mx
}

\begin{abstract}
In this work we will solve the problem of expression of the sum of two given elements of a finite field, as power of the primitive element of the field. We obtain a reduced table of the Zech's logarithm from our proposal that relate the Zech'slogarithm with the partition of the exponents of the powers of elements over finite field $\boldsymbol{G} \boldsymbol{F}\left(\boldsymbol{p}^{\boldsymbol{n}}\right)$ in $\mathrm{p}$-cyclotomic cosets modulo $\left(\boldsymbol{p}^{n}-\mathbf{1}\right)$. This reduces, in a significant way, the quantity of information to store and it facilitates its use in several cryptographic algorithms, specifically in asimetric cryptography. It is illustrated the computationof the Zech'slogarithm of any element thatdoesn't appear in the obtained reduced table.
\end{abstract}

\section{Indexingterms/Keywords}

Finite field; Zech's logarithm; cyclotomiccoset.

\section{Academic Discipline And Sub-Disciplines}

Discipline: Matthematics, Computer Science.Sub-Disciplines: Cryptology, Theoretical Computer Science.

\section{MATHEMATICS SUBJECTCLASSIFICATION}

\author{
MSC 2010:12E30, 11T22, 11T71
}

\section{INTRODUCTION}

The study of finite fields has made great progress in recent years because they are applied in areas as diverse as cryptography [1, 2, 3], coding theory [4, 5, 6], among other areas [7, 8]. By working with finite fields, arithmetic operations with its elements are performed, so you need to have efficient algorithms to perform such operations, this being a major problem today.

Considering that multiplication of elements of a finite field is a polynomial multiplication it is convenient express the elements of the field as powers of a primitive element, so that the multiplication of them is reduced to the sum of their exponents. Also is necessary have a procedure to perform efficiently the sum of two elements of the field expressed as a power of the primitive element, since this operation under the above condition is not trivial. To solve this the so-called Zech's logarithm table [6] is used.

Another related problem with the above is when there are two binary finite fields $G F\left(2^{n}\right)$ and $G F\left(2^{m}\right)$ with primitive elements is $\alpha$ and $\beta$ respectively (GF, denote Galois Field). All elements of $G F\left(2^{n}\right)$ and $G F\left(2^{m}\right)$ can be expressed as powers of $\alpha$ and $\beta$ respectively. The elements of both fields can also be expressed as a binary digit sequence of $\mathrm{n}$ and $\mathrm{m}$ respectively. If we add $m-n$ bits to the right of the elements $\alpha^{i}$ we obtain $2^{m-n}$ sequences of $m$ digits .An interesting question is ¿How to determine wichpower of the primitive element $\beta$, over the field $G F\left(2^{m}\right)$, representthese new sequences?

In this paper a proposal that significantly reduces the Zech's logarithm table is presented.

\section{APPROACH PROBLEMS}

\section{Problem 1}

Let the finite field $G F\left(2^{n}\right)$ which is an algebraic extension of degree $\mathrm{n}$ of the prime field $G F(p)$.We want to find the pairs $(i, j)$ satisfying,

$$
1+\alpha^{i}=\alpha^{j}
$$

Considering that in any finite field of characteristic $p$ [8].

$$
\begin{aligned}
& \left(1+\alpha^{i}\right)^{\mathrm{p}}=\left(\alpha^{j}\right)^{p} \Rightarrow 1+\alpha^{i p}=\alpha^{j p} \\
& \left(1+\alpha^{i}\right)^{\mathrm{p}^{k}}=\left(\alpha^{j}\right)^{\mathrm{p}^{k}} \Rightarrow 1+\alpha^{i p^{k}}=\alpha^{j p^{k}}
\end{aligned}
$$


is satisfied. Then if the pair $(i, j)$ satisfies the relation (1) then the pair $\left(i \times p^{k}, j \times p^{k}\right)$ also satisfies. The $i \times p^{k} \mathrm{y} j \times$ $p^{k}$ elements are reduced modulo $p^{\mathrm{n}}-1$. How to find these pairs?

\section{Problem 2}

Suppose we have the finite field $G F(q)=G F\left(p^{n}\right)$ where $p$ is a prime number and $n$ is a positive integer. Let $\alpha$ a primitive element of the field $G F\left(p^{n}\right)$.If there are two elements of a finite field expressed as powers of the primitive element, how to express their sum as a power of the primitive element?.

In other words. If $i, j, k$ are non-negative integers such numbers that $i \neq j \neq k y i \neq j \neq 0$,if $\alpha^{i}+\alpha^{j}=\alpha^{k}$. How to find $k$ ?

If in the expression $\alpha^{i}+\alpha^{j}$ we extract the common factor under the powers we can reduce the problem to the previous case.

Suppose that $i<j$, we have, with $v=(j-i) \bmod \left(\mathrm{p}^{n}-1\right)$,

$$
\alpha^{i}+\alpha^{j}=\alpha^{i}\left(1+\alpha^{v}\right)
$$

then,

$$
1+\alpha^{v}=\alpha^{r} \rightarrow \alpha^{i} \cdot \alpha^{r}=\alpha^{(i+r) \bmod \left(\mathrm{p}^{n}-1\right)}
$$

To answer this questions these problems for $G F\left(p^{n}\right)$ and $n=\overline{2,8}$ fields were resolved. The results enabled us to detect regularities that allow us to build a reduced table ofZech'slogarithm, considering the $\mathrm{p}$-cyclotomiccosetsmodulo $\mathrm{p}^{n}-1$.

Let $\alpha$ a generator element of the multiplicative group of the field $G F\left(p^{n}\right)$, the product of two elements of the finite field are represented by $\alpha^{i} \cdot \alpha^{j}=\alpha^{(i+j) \bmod \left(\mathrm{p}^{n}-1\right)}$ and the addition of the elements of the finite field expressed as powers ofprimitive element is facilitated if the calledZech'slogarithm table is built [6], where for each integer $i, 0 \leq i \leq p^{n-2}$, the integer $j=$ $z(i)$ such that

$$
1+\alpha^{i}=\alpha^{j}=\alpha^{\mathrm{z}(\mathrm{i})}
$$

is determined. Then,

$$
\alpha^{i}+\alpha^{j}=\alpha^{i} \cdot\left(1+\alpha^{v}\right)=\alpha^{i} \cdot \alpha^{\mathrm{z}(\mathrm{j}-\mathrm{i})}=\alpha^{\mathrm{i}+\mathrm{z}(\mathrm{v})},
$$

where $z(v)$ is taken from the Zech's logarithm table.

Given (4) and the analysis in the problem statement,the expressions (2) and (3) can be rewritten as follows

$$
\begin{gathered}
\left(1+\alpha^{i}\right)^{\mathrm{p}}=\alpha^{\mathrm{z}(\mathrm{i})}{ }^{p} \Rightarrow 1+\alpha^{i p}=\alpha^{\mathrm{p} \times \mathrm{z}(\mathrm{i})} \\
\left(1+\alpha^{i}\right)^{\mathrm{p}^{k}}=\alpha^{\mathrm{z}(\mathrm{i})} p^{p^{k}} \Rightarrow 1+\alpha^{i p^{k}}=\alpha^{\mathrm{p}^{k} \times \mathrm{z}(\mathrm{i})}
\end{gathered}
$$

\section{RELATIONSHIP BETWEEN THEZECH'S LOGARITHMAND THE CYCLOTOMIC COSETS}

We began recall that the q-cyclotomiccosets[9] [10] of $S$ modulo $n$ is defined by the set $C_{s}=\left\{s, s q, s q^{2}, \cdots, s q^{r-1}\right\}$, where $r$ is the smallest positive integer such that $s q^{\mathrm{r}} \equiv s(\bmod n)$. In particular, the2-cyclotomiccoset[9] [10]modulon is the set $C_{s}=$ $\left\{s, s 2, s 2^{2}, \cdots, s 2^{r-1}\right\}$, where $r$ is the smallest positive integer such that $s 2^{r} \equiv s(\bmod n)$.

We noted earlier thatif the pair $(i, j)$ satisfies the relation(1) then the pair $\left(i \times p^{k}, j \times p^{k}\right)$ also satisfies it .The expression $i \times p^{k}\left(\bmod \left(\mathrm{p}^{n}-1\right)\right)$ where $i \in[0, r]$ represents all the elements that arein the same $p$-cyclotomiccosetmodulo $\mathrm{p}^{n}-1$.Thereforeknowing the Zech'slogarithm of an integer $i$ we can find the Zech's logarithm ofallintegers of the form $i \times p^{k}\left(\bmod \left(\mathrm{p}^{n}-1\right)\right)$.

We illustrate the above taking a particular finite field.

Let the finite field $G F\left(2^{8}\right)$ we take primitive polynomial $x^{8}+x^{4}+x^{3}+x^{2}+1$ as the defining polynomial. Let $\alpha$ be a root of this polynomialand hence a primitive field element.

The2-cyclotomiccosets [9] modulo 255 are as follows

$$
\begin{aligned}
& C_{0}=\{0\} \\
& C_{1}=\{1,2,4,8,16,32,64,128\} \\
& C_{3}=\{3,6,12,24,48,96,192,129\} \\
& C_{5}=\{5,10,20,40,80,160,65,130\} \\
& C_{7}=\{7,14,28,56,112,224,193,131\} \\
& C_{9}=\{9,18,36,72,144,33,66,132\} \\
& C_{11}=\{11,22,44,88,176,97,194,133\}
\end{aligned}
$$

$$
\begin{aligned}
& C_{13}=\{13,26,52,104,208,161,67,134\} \\
& C_{15}=\{15,30,60,120,240,225,195,135\} \\
& C_{17}=\{17,34,68,136\} \\
& C_{19}=\{19,38,76,152,49,98,196,137\} \\
& C_{21}=\{21,42,84,168,81,162,69,138\} \\
& C_{23}=\{23,46,92,184,113,226,197,139\} \\
& C_{25}=\{25,50,100,200,145,35,70,140\}
\end{aligned}
$$




$$
\begin{aligned}
& C_{27}=\{27,54,108,216,177,99,198,141\} \\
& C_{29}=\{29,58,116,232,209,163,71,142\} \\
& C_{31}=\{31,62,124,248,241,227,199,143\} \\
& C_{37}=\{37,74,148,41,82,164,73,146\} \\
& C_{39}=\{39,78,156,57,114,228,201,147\} \\
& C_{43}=\{43,86,172,89,178,101,202,149\} \\
& C_{45}=\{45,90,180,105,210,165,75,150\} \\
& C_{47}=\{47,94,188,121,242,229,203,151\} \\
& C_{51}=\{51,102,153,204\} \\
& C_{53}=\{53,106,212,169,83,166,77,154\} \\
& C_{55}=\{55,110,220,185,115,230,205,155\}
\end{aligned}
$$

$$
\begin{aligned}
& C_{59}=\{59,118,236,217,179,103,206,157\} \\
& C_{61}=\{61,122,244,233,211,167,79,158\} \\
& C_{63}=\{63,126,252,249,243,231,207,159\} \\
& C_{85}=\{85,170\} \\
& C_{87}=\{87,174,93,186,117,234,213,171\} \\
& C_{91}=\{91,182,109,218,181,107,214,173\} \\
& C_{95}=\{95,190,125,250,245,235,215,175\} \\
& C_{111}=\{111,222,189,123,246,237,219,183\} \\
& C_{119}=\{119,238,221,187\} \\
& C_{127}=\{127,254,253,251,247,239,223,191\}
\end{aligned}
$$

The cosets $\mathrm{C}_{17}, \mathrm{C}_{51}, \mathrm{C}_{85}, \mathrm{C}_{119}$ are the regular cyclotomiccosets [10] .In this case we have 35 cyclotomiccosetsdenoted by $\mathrm{C}_{\mathrm{i} w h e r e} i$ is the coset leaders $0 \leq i \leq 127$. So, $i$ is a positive integer that takes values in the range from 0 to $\frac{\mathrm{p}^{n}-1}{2}$.

Thetable of Zech's logarithm for the field $G F\left(2^{8}\right)$ with defining polynomial $x^{8}+x^{4}+x^{3}+x^{2}+1$,grouping the values of $j$ according to the cyclotomic coset that they belong, appears in the Annex.

Here are some examples of computation the sum of two elements of a finite field using Zech's logarithm table.

\section{Example1:}

a. $\quad \alpha^{7}+\alpha^{47}=\alpha^{7} \cdot\left(1+\alpha^{40}\right)=\alpha^{7} \cdot\left(1+\alpha^{5 \times 2^{3}}\right)=\alpha^{7} \cdot\left(1+\alpha^{5}\right)^{2^{3}}=\alpha^{7} \cdot \alpha^{z(5) \times 8(\bmod 255)}$

$$
=\alpha^{7} \cdot \alpha^{138 \times 8(\bmod 255)}=\alpha^{7+84}=\alpha^{91}
$$

b. $\quad \alpha^{37}+\alpha^{103}=\alpha^{37} \cdot\left(1+\alpha^{66}\right)=\alpha^{37} \cdot\left(1+\alpha^{33 \times 2}\right)=\alpha^{37} \cdot\left(1+\alpha^{33}\right)^{2}=\alpha^{37} \cdot \alpha^{z(33) \times 2(\bmod 255)}$

$=\alpha^{37} \cdot \alpha^{15 \times 2(\bmod 255)}=\alpha^{67}$

c. $\quad \alpha^{219}+\alpha^{135}=\alpha^{135} \cdot\left(1+\alpha^{84}\right)=\alpha^{135} \cdot\left(1+\alpha^{21 \times 2^{2}}\right)=\alpha^{135} \cdot\left(1+\alpha^{21}\right)^{2^{2}}=\alpha^{135} \cdot \alpha^{z(21) \times 4(\bmod 255)}$

$=\alpha^{135} \cdot \alpha^{40}=\alpha^{175}$

Now we buildthe table of theZech's logarithm given the partition of the set of non-negative integers $\{0,1,2,3, \cdots, 254\}$ in $2-$ cyclotomiccosetsmodulo255. This allows us not store the entire table but only the values of the Zech's logarithm for the coset leaders.

The Zech's logarithm for the finite field $G F\left(2^{8}\right)$ with defining primitive polynomial $x^{8}+x^{4}+x^{3}+x^{2}+1$, considering onlythe coset leaders, is showed in the following table.

Table 1: 2-cyclotomiccosets modulo 255

\begin{tabular}{|c|c|c|c|c|c|c|c|}
\hline $\mathbf{j}$ & $\mathbf{z}(\mathbf{j})$ & $\mathbf{j}$ & $\mathbf{z}(\mathbf{j})$ & $\mathbf{j}$ & $\mathbf{z}(\mathbf{j})$ & $\mathbf{j}$ & $\mathbf{z}(\mathbf{j})$ \\
\hline 0 & $\infty$ & 15 & 33 & 39 & 106 & 63 & 55 \\
\hline & & 17 & 68 & 43 & 121 & 85 & 170 \\
\hline 1 & 25 & 19 & 92 & 45 & 31 & 87 & 167 \\
\hline 3 & 223 & 21 & 10 & 47 & 101 & 91 & 209 \\
\hline 5 & 138 & 23 & 196 & 51 & 238 & 95 & 176 \\
\hline 7 & 112 & 25 & 1 & 53 & 147 & 111 & 246 \\
\hline 9 & 120 & 27 & 104 & 55 & 63 & 119 & 153 \\
\hline 11 & 245 & 31 & 45 & 59 & 82 & 127 & 12 \\
\hline 13 & 99 & 37 & 179 & 61 & 186 & & \\
\hline
\end{tabular}

Using the table of coset leaders to calculate Zech's logarithm of a value of $d$ that is not on the table. The following cases may arise:

1. The number $d$ is the product of a power of the characteristic field and an odd number which is a coset leader. 
2. The number $d$ is an odd number that is not a coset leader.

3. The number $d$ is the product of a power of the characteristic field and an odd number which that is not a coset leader.

\section{Case 1:}

If $d=i \times p^{k}$ then $z(d)=z(i) \times p^{k}\left(\bmod \left(p^{n}-1\right)\right)$

\section{Case 2:}

We add $\left(\mathbf{p}^{n}-\mathbf{1}\right)$ to $\boldsymbol{d}$ and expressed as the product of the largest possible power of thecharacteristicfieldand an odd number,

$$
d+\left(\mathrm{p}^{n}-1\right)=d_{1} \rightarrow d_{1}=d_{2} \times p^{k_{1}} \rightarrow d_{2}+\left(\mathrm{p}^{n}-1\right)=d_{3} \rightarrow d_{3}=d_{4} \times p^{k_{2}} \rightarrow \cdots
$$

Suppose that for $\boldsymbol{r}$ is obtained :

$$
d_{r-1}=d_{r} \times p^{\frac{k_{r}}{2}}
$$

where $d_{r}$ is a coset leader.Stop the process and

$$
\begin{aligned}
& d=d_{r} \times p^{k_{1}} \times p^{k_{2}} \times \cdots \times p^{\frac{k_{r}}{2}}=d_{r} \times p^{k} \text { being } k=k_{1}+k_{2}+\cdots+\frac{k_{r}}{2}, \text { with } k_{1}, k_{2}, \cdots, \frac{k_{r}}{2} \in[0, n-1] . \\
& z(d)=Z\left(d_{r}\right) \times p^{k}\left(\bmod \left(p^{n}-1\right)\right)=Z(i) \times p^{k}\left(\bmod \left(p^{n}-1\right)\right) \text { for same cosetleader } i .
\end{aligned}
$$

\section{Case 3:}

If $\boldsymbol{d}=\boldsymbol{d}_{\mathbf{1}} \times \boldsymbol{p}^{\boldsymbol{k}_{\mathbf{1}}}$, we proceed to $\boldsymbol{d}_{\mathbf{1}}$ similarly to Case 2 .

\section{Example 2:}

Compute in $G F\left(2^{8}\right)$,

a) $z(168)=z(21) \times 2^{3}(\bmod 255)=10 \times 8=80$

b) $z(197)$

197 is an odd number that is not a coset leader

$197+255=452 \rightarrow 452=113 \times 2^{2} \rightarrow 113+255=368 \rightarrow 368=23 \times 2^{4}$

As23 is a cosetleader then

$197 \equiv 23 \times 2^{2} \times 2^{4}(\bmod 255)=23 \times 2^{6}(\bmod 255) \equiv 226$

$z(197)=z\left(23 \times 2^{6}(\bmod 255)\right)=z(23) \times 2^{6}(\bmod 255)=196 \times 64(\bmod 255) \equiv 49$

c) $z(228)$

$228=57 \times 2^{2} \rightarrow 57+255=312 \rightarrow 312=39 \times 2^{3}$

39 is a cosetleader

$228 \equiv 39 \times 2^{2} \times 2^{3}(\bmod 255)=39 \times 2^{5}(\bmod 255)$

$z(228) \equiv z(39) \times 2^{5}(\bmod 255)=106 \times 32(\bmod 255)=77$

\section{CONCLUSIONS}

In this paper a modification to the table of Zech's logarithm for the field $G F\left(2^{8}\right)$, applicable to any given field, consisting of a considerable reduction of the number of elements to be stored is proposed. For this the called p-cyclotomic cosets were used. This result is essential to perform the addition operation between two elements of a finite field represented as powers of a primitive element of this field. It also has various applications in cryptography, especially in the implementation of cryptographic algorithms.

\section{REFERENCES}

1. Didier F., M. and Laigle-ChapuyY.2007. Finding low-weight polynomial multiples using discrete logarithm.

2. Johansson T.2014.Low weight polynomials in crypto. 
3. Kiihn G. J. and W. Penzhorn T. 1994. Using Zech's Logarithm to Find Low-Weight Parity Checks for Linear Recurring Sequences. Communications and cryptography.

4. Elsenhans A.-S., KohnertA. and Wassermann A. 2010. Construction of Codes for Network Coding. In Proceedings of the 19th International Symposium on Mathematical Theory of Networks and Systems - MTNS 2010.

5. LiJ. 2004. Combinatorially Designed LDPC Codes Using Zech Logarithms and Congruential Sequences.Coding, Cryptographyand Combinatorics.

6. Huber K.1990. Some Comments on Zech's Logarithms. IEEE Transactions on Information Theory.

7. Meyer-Baese U. 2004. Digital Signal Processing with Field Programmable Gate Arrays. SecondEdition.

8. MullenG. L.and Panario D.2013. Handbook of Finite Fields, CRC Pres.Taylor\& Francis Group.

9. Golomb S. W. 1981. Shift Register Sequences, Aegean Park Press. Laguna Hills, CA, USA.

10. Rani M. J. 2013 Cyclic Codes of length N over GF(q)q- ciclotomiccosets modulo $N$ and application of Burnside's lemma. InternationalJournal of Scientific and Research Publications.

\section{ANEX}

Table of the Zech'slogarithm forthe finite field $G F\left(2^{8}\right)$ with definingprimitive polynomial $x^{8}+x^{4}+x^{3}+x^{2}+1$

$1+\alpha^{i}=\alpha^{\mathrm{z}(\mathrm{i})}$

\begin{tabular}{|c|c|c|c|c|c|c|c|}
\hline$i$ & $z(i)$ & $i$ & $z(i)$ & $i$ & $z(i)$ & $i$ & $z(i)$ \\
\hline 1 & 25 & 3 & 223 & 5 & 138 & 7 & 112 \\
\hline 2 & 50 & 6 & 191 & 10 & 21 & 14 & 224 \\
\hline 4 & 100 & 12 & 127 & 20 & 42 & 28 & 193 \\
\hline 8 & 200 & 24 & 254 & 40 & 84 & 56 & 131 \\
\hline 16 & 145 & 48 & 253 & 80 & 168 & 112 & 7 \\
\hline 32 & 35 & 96 & 251 & 160 & 81 & 224 & 14 \\
\hline 64 & 70 & 192 & 247 & 65 & 162 & 193 & 28 \\
\hline 128 & 140 & 129 & 239 & 130 & 69 & 131 & 56 \\
\hline$i$ & $z(i)$ & $i$ & $\bar{z}(\boldsymbol{i})$ & $i$ & $z(i)$ & $\bar{i}$ & $\mathbf{z}(\boldsymbol{i})$ \\
\hline 9 & 120 & 11 & 245 & 13 & 99 & 15 & 33 \\
\hline 18 & 240 & 22 & 235 & 26 & 198 & 30 & 66 \\
\hline 36 & 225 & 44 & 215 & 52 & 141 & 60 & 132 \\
\hline 72 & 195 & 88 & 175 & 104 & 27 & 120 & 68 \\
\hline 144 & 135 & 176 & 95 & 208 & 54 & 240 & 136 \\
\hline 33 & 15 & 97 & 190 & 161 & 108 & 225 & 17 \\
\hline 66 & 30 & 194 & 125 & 67 & 216 & 195 & 34 \\
\hline 132 & 60 & 133 & 250 & 134 & 177 & 135 & 68 \\
\hline 17 & 68 & 51 & 238 & 85 & 170 & 119 & 153 \\
\hline 34 & 136 & 102 & 221 & 170 & 85 & 238 & 51 \\
\hline 68 & 17 & 204 & 187 & $\infty$ & 0 & 221 & 102 \\
\hline 136 & 34 & 153 & 119 & 0 & $\infty$ & 187 & 204 \\
\hline 19 & 92 & 21 & 10 & 23 & 196 & 25 & 1 \\
\hline 38 & 184 & 42 & 20 & 46 & 137 & 50 & 2 \\
\hline
\end{tabular}



Journal of Advances in Mathematics

\begin{tabular}{|c|c|c|c|c|c|c|c|}
\hline 76 & 113 & 84 & 40 & 92 & 19 & 100 & 4 \\
\hline 152 & 138 & 168 & 80 & 184 & 38 & 200 & 8 \\
\hline 49 & 197 & 81 & 160 & 113 & 76 & 145 & 16 \\
\hline 98 & 139 & 162 & 65 & 226 & 152 & 35 & 32 \\
\hline 196 & 23 & 69 & 130 & 197 & 49 & 70 & 64 \\
\hline 137 & 46 & 138 & 5 & 139 & 98 & 140 & 128 \\
\hline 27 & 104 & 29 & 181 & 31 & 45 & 37 & 179 \\
\hline 54 & 208 & 58 & 107 & 62 & 90 & 74 & 103 \\
\hline 108 & 161 & 116 & 214 & 124 & 180 & 148 & 206 \\
\hline 216 & 67 & 232 & 173 & 248 & 105 & 41 & 157 \\
\hline 177 & 134 & 209 & 91 & 241 & 210 & 82 & 59 \\
\hline 99 & 13 & 163 & 182 & 227 & 165 & 164 & 179 \\
\hline 198 & 26 & 71 & 109 & 199 & 75 & 73 & 103 \\
\hline 141 & 52 & 142 & 218 & 143 & 150 & 146 & 206 \\
\hline 39 & 106 & 43 & 121 & 45 & 31 & 47 & 101 \\
\hline 78 & 212 & 86 & 242 & 90 & 62 & 94 & 202 \\
\hline 156 & 169 & 172 & 229 & 180 & 124 & 188 & 149 \\
\hline 57 & 83 & 89 & 203 & 105 & 248 & 121 & 43 \\
\hline 114 & 166 & 178 & 151 & 210 & 241 & 242 & 86 \\
\hline 228 & 77 & 101 & 47 & 165 & 227 & 229 & 172 \\
\hline 201 & 154 & 202 & 94 & 75 & 199 & 203 & 89 \\
\hline 147 & 53 & 149 & 188 & 150 & 143 & 151 & 178 \\
\hline 53 & 147 & 55 & 63 & 59 & 82 & 61 & 186 \\
\hline 106 & 39 & 110 & 126 & 118 & 164 & 122 & 117 \\
\hline 212 & 78 & 220 & 252 & 236 & 73 & 244 & 234 \\
\hline 169 & 156 & 185 & 249 & 217 & 146 & 233 & 213 \\
\hline 83 & 57 & 115 & 243 & 179 & 37 & 211 & 171 \\
\hline 166 & 114 & 230 & 231 & 103 & 74 & 167 & 87 \\
\hline 77 & 228 & 205 & 207 & 206 & 148 & 79 & 174 \\
\hline 154 & 201 & 155 & 159 & 157 & 41 & 158 & 93 \\
\hline
\end{tabular}


I S S N $2347-1921$

Volume $12 \mathrm{Number} 07$ Journal of Advances in Mathematics

\begin{tabular}{|c|c|c|c|c|c|c|c|}
\hline$i$ & $z(i)$ & $i$ & $z(i)$ & $i$ & $z(i)$ & $i$ & $\mathbf{z}(i)$ \\
\hline 87 & 167 & 91 & 209 & 95 & 176 & 11 & 246 \\
\hline 174 & 79 & 182 & 163 & 190 & 97 & 222 & 237 \\
\hline 93 & 158 & 109 & 71 & 125 & 194 & 189 & 219 \\
\hline 186 & 61 & 218 & 142 & 250 & 133 & 123 & 183 \\
\hline 117 & 122 & 181 & 29 & 245 & 11 & 246 & 111 \\
\hline 234 & 244 & 107 & 58 & 235 & 22 & 237 & 222 \\
\hline 213 & 233 & 214 & 116 & 215 & 44 & 219 & 189 \\
\hline 171 & 211 & 173 & 232 & 175 & 88 & 183 & 123 \\
\hline 127 & 12 & 253 & 48 & 247 & 192 & 223 & 3 \\
\hline 254 & 24 & 251 & 96 & 239 & 129 & 191 & 6 \\
\hline
\end{tabular}

